\title{
CONVOLUTIONS OF PLANAR HARMONIC CONVEX MAPPINGS
}

\author{
MICHAEL DORFF
}

\begin{abstract}
Ruscheweyh and Sheil-Small proved that convexity is preserved under the convolution of univalent analytic mappings in $K$. However, when we consider the convolution of univalent harmonic convex mappings in $K_{H}^{O}$, this property does not hold. In fact, such convolutions may not be univalent. We establish some results concerning the convolution of univalent harmonic convex mappings provided that it is locally univalent. In particular, we show that the convolution of a right half-plane mapping in $K_{H}^{O}$ with either another right halfplane mapping or a vertical strip mapping in $K_{H}^{O}$ is convex in the direction of the real axis. Further, we give a condition under which the convolution of a vertical strip mapping in $K_{H}^{O}$ with itself will be convex in the direction of the real axis.
\end{abstract}

\section{INTRODUCTION}

Let $\mathbb{D}$ be the unit disk. We will consider the family of complex-valued harmonic functions $f=u+i v$ defined in $\mathbb{D}$, where $u$ and $v$ are real harmonic in $\mathbb{D}$. Such functions can be expressed as $f=h+\bar{g}$, where

$$
h(z)=z+\sum_{n=2}^{\infty} a_{n} z^{n} \text { and } g(z)=\sum_{n=1}^{\infty} b_{n} z^{n}
$$

are analytic in $\mathbb{D}$. The harmonic $f=h+\bar{g}$ is locally one-to-one and sensepreserving in $\mathbb{D}$ if and only if

$$
\left|g^{\prime}(z)\right|<\left|h^{\prime}(z)\right|, \forall z \in \mathbb{D} \text {. }
$$

In such case, we say that $f$ is locally univalent. Let $S_{H}$ be the class of complexvalued, harmonic, sense-preserving, univalent functions $f$ in $\mathbb{D}$, normalized so that $f(0)=0$ and $f_{z}(0)=1$. The classical family $S$ of analytic univalent, normalized functions on $\mathbb{D}$ is the subclass of $S_{H}$ in which $b_{k}=0$ for all $k$. Let $K_{H}, S_{H}^{*}$, and $C_{H}$ be the subclasses of $S_{H}$ mapping $\mathbb{D}$ onto convex, starlike, and close-to-convex domains, just as $K, S^{*}$, and $C$ are the subclasses of $S$ mapping $\mathbb{D}$ onto these respective domains. Finally, let $S_{H}^{O}$ denote the subclass of $S_{H}$ for which $f_{\bar{z}}(0)=\overline{b_{1}}=0$, and $K_{H}^{O}$ and $C_{H}^{O}$ be the subclasses of $S_{H}^{O}$ of convex and close-to-convex mappings, respectively.

For analytic functions $f(z)=z+\sum_{n=2}^{\infty} a_{n} z^{n}$ and $F(z)=z+\sum_{n=2}^{\infty} A_{n} z^{n}$, their convolution (or Hadamard product) is defined as $f * F=z+\sum_{n=2}^{\infty} a_{n} A_{n} z^{n}$. In the

Date: March 2000.

1991 Mathematics Subject Classification. Primary 30C45.

Key words and phrases. harmonic mappings, convolutions, convex. 
harmonic case, with

$$
\begin{aligned}
& f=h+\bar{g}=z+\sum_{n=2}^{\infty} a_{n} z^{n}+\sum_{n=1}^{\infty} \bar{b}_{n} \bar{z}^{n} \text { and } \\
& F=H+\bar{G}=z+\sum_{n=2}^{\infty} A_{n} z^{n}+\sum_{n=1}^{\infty} \bar{B}_{n} \bar{z}^{n},
\end{aligned}
$$

define the harmonic convolution as

$$
f \widetilde{*} F=h * H+\overline{g * G}=z+\sum_{n=2}^{\infty} a_{n} A_{n} z^{n}+\sum_{n=1}^{\infty} \overline{b_{n} B_{n}} \bar{z}^{n},
$$

Clunie and Sheil-Small [2] posed the question for what harmonic functions $\varphi$ is $\varphi \widetilde{*} f$ in $K_{H}$, where $f \in K_{H}$ ? This question was partially answered by Ruscheweyh and Salinas [8]. In this paper, we provide some further results related to this question.

\section{BACKGROUND RESULTS}

In [1], [3], and [6], explicit descriptions are given for half-plane and strip mappings. Specifically, the collection of functions $f=h+\bar{g} \in S_{H}^{O}$ that map $\mathbb{D}$ onto the right half-plane, $R=\{w: \operatorname{Re}(w)>-1 / 2\}$, have the form

$$
h(z)+g(z)=\frac{z}{1-z}
$$

and those that map $\mathbb{D}$ onto the vertical strip, $\Omega_{\alpha}=\left\{w: \frac{\alpha-\pi}{2 \sin \alpha}<\operatorname{Re}(w)<\frac{\alpha}{2 \sin \alpha}\right\}$, where $\frac{\pi}{2} \leq \alpha<\pi$, have the form

$$
h(z)+g(z)=\frac{1}{2 i \sin \alpha} \log \left(\frac{1+z e^{i \alpha}}{1+z e^{-i \alpha}}\right) .
$$

Note that a function in $S_{H}^{O}$ satisfying (2) maps $\mathbb{D}$ onto $\Omega_{\alpha}$, or onto the convex hull of three points (one of which may be the point at infinity) on the boundary of $\Omega_{\alpha}$. In other words, the image of $\mathbb{D}$ may be a vertical strip, a halfstrip, a trapezium, or a triangle.

In proving our theorems we will need a few known results. The first is Clunie and Sheil-Small's shear construction theorem ([2], Theorem 5.3).

Theorem 1. A harmonic function $f=h+\bar{g}$ locally univalent in $\mathbb{D}$ is a univalent mapping of $\mathbb{D}$ onto a domain convex in the direction of the real axis if and only if $h-g$ is an analytic univalent mapping of $\mathbb{D}$ onto a domain convex in the direction of the real axis.

Next, there is a useful remark by Pommerenke [7] concerning analytic mappings convex in one direction. Using a particular case of this, we have the following result.

Theorem 2. Let $f$ be an analytic function in $\mathbb{D}$ with $f(0)=0$ and $f^{\prime}(0) \neq 0$, and let

where $\theta \in \mathbb{R}$. If

$$
\varphi(z)=\frac{z}{\left(1+z e^{i \theta}\right)\left(1+e^{-i \theta}\right)}
$$

$$
\operatorname{Re}\left\{\frac{z f^{\prime}(z)}{\varphi(z)}\right\}>0, \forall z \in \mathbb{D},
$$

then $f$ is convex in the direction of the real axis. 
Finally, we state a result by Ruscheweyh and Sheil-Small ([9], Lemma 2.7) concerning convolution of analytic functions.

Theorem 3. Let $\varphi$ and $G(z)$ be analytic in $\mathbb{D}$ with $\varphi(0)=G(0)=0$. If $\varphi$ is convex and $G$ is starlike, then for each function $F(z)$ analytic in $\mathbb{D}$ and satisfying $\operatorname{Re} F(z)>0$, we have

$$
\operatorname{Re} \frac{(\varphi * F G)(z)}{(\varphi * G)(z)}>0, \forall z \in \mathbb{D}
$$

\section{MAin Results}

For the convolution of analytic functions, if $f_{1}, f_{2} \in K$, then $f_{1} * f_{2} \in K$. Also, the right half-plane mapping, $\frac{z}{1-z}$, acts as the convolution identity. In the harmonic case, there are infinitely many right half-plane mappings and the harmonic convolution of one of these right half-plane mappings with a function $f \in K_{H}^{O}$ may not preserve the properties of $f$, such as convexity or even univalence. This fact is seen in the following example.

Example 4. If $l_{0}=h_{0}+\bar{g}_{0} \in K_{H}^{O}$, where

$$
h_{0}(z)=\frac{z-\frac{1}{2} z^{2}}{(1-z)^{2}}=z+\sum_{n=2}^{\infty} \frac{1+n}{2} z^{n} \text { and } g_{0}(z)=\frac{-\frac{1}{2} z^{2}}{(1-z)^{2}}=\sum_{n=2}^{\infty} \frac{1-n}{2} z^{n},
$$

is the right half-plane mapping presented in [2], and if $f_{1}=h_{1}+\bar{g}_{1} \in K_{H}^{O}$ is the mapping, described by Duren [5], onto a 6-gon, where

$$
h_{1}(z)=z+\sum_{n=1}^{\infty} \frac{1}{6 n+1} z^{6 n+1} \text { and } g_{1}(z)=\sum_{n=1}^{\infty} \frac{-1}{6 n-1} z^{6 n-1}
$$

(see [4] for these representations of $h_{1}$ and $\left.g_{1}\right)$. Then $l_{0} \widetilde{*} f_{1} \notin K_{H}^{O}$, because $\mid\left(g_{0}(z) *\right.$ $\left.g_{1}(z)\right)^{\prime} /\left(h_{0}(z) * h_{1}(z)\right)^{\prime}|=| z^{4}\left(2+z^{6}\right) /\left(1+2 z^{6}\right) \mid \nless 1, \forall z \in \mathbb{D}$.

However, the next theorem guarantees that the harmonic convolution of a right half-plane mapping with another right half-plane mapping will at least be convex in the direction of the real axis as long as the convolution is sense-preserving.

Theorem 5. Let $f_{1}=h_{1}+\bar{g}_{1}, f_{2}=h_{2}+\bar{g}_{2} \in K_{H}^{O}$ be right half-plane mappings. If $f_{1} \widetilde{*} f_{2}$ satisfies (1), then $f_{1} \widetilde{*} f_{2} \in C_{H}^{O}$ and is convex in the direction of the real axis.

Proof. For any right half-plane mapping $f=h+\bar{g} \in K_{H}^{O}$, recall that

$$
h+g=\frac{z}{1-z} .
$$

Hence,

$$
\begin{aligned}
& h_{2}-g_{2}=\left(h_{1}+g_{1}\right) *\left(h_{2}-g_{2}\right)=h_{1} * h_{2}-h_{1} * g_{2}+h_{2} * g_{1}-g_{1} * g_{2} \\
& h_{1}-g_{1}=\left(h_{1}-g_{1}\right) *\left(h_{2}+g_{2}\right)=h_{1} * h_{2}+h_{1} * g_{2}-h_{2} * g_{1}-g_{1} * g_{2} .
\end{aligned}
$$

Thus,

$$
h_{1} * h_{2}-g_{1} * g_{2}=\frac{1}{2}\left[\left(h_{1}-g_{1}\right)+\left(h_{2}-g_{2}\right)\right] .
$$

We will now show that $\left(h_{1}-g_{1}\right)+\left(h_{2}-g_{2}\right)$ is convex in the direction of the real axis. 
If $f=h+\bar{g} \in K_{H}^{O}$ is a right half-plane mappings with dilatation $\omega=g^{\prime} / h^{\prime}$, then

$$
h^{\prime}-g^{\prime}=\left(h^{\prime}+g^{\prime}\right)\left(\frac{h^{\prime}-g^{\prime}}{h^{\prime}+g^{\prime}}\right)=\left(h^{\prime}+g^{\prime}\right)\left(\frac{1-\omega}{1+\omega}\right)=\frac{p(z)}{(1-z)^{2}},
$$

where $\operatorname{Re}\{p(z)\}>0, \forall z \in \mathbb{D}$. So, letting $\varphi(z)=z /(1-z)^{2} \in S^{*}$, we have

$$
\begin{aligned}
\operatorname{Re}\left\{\frac{z\left[\left(h_{1}^{\prime}-g_{1}^{\prime}\right)+\left(h_{2}^{\prime}-g_{2}^{\prime}\right)\right]}{\varphi}\right\} & =\operatorname{Re}\left\{\frac{\frac{z}{(1-z)^{2}}\left[p_{1}(z)+p_{2}(z)\right]}{\frac{z}{(1-z)^{2}}}\right\} \\
& =\operatorname{Re}\left\{p_{1}(z)+p_{2}(z)\right\}>0 .
\end{aligned}
$$

Therefore, by Theorem 2 and eq. (3), $h_{1} * h_{2}-g_{1} * g_{2}$ is convex in the direction of the real axis.

Finally, since we assumed that $f_{1} \widetilde{*} f_{2}$ is locally univalent, we apply Theorem 1 to get that $f_{1} \approx f_{2}=h_{1} * h_{2}-g_{1} * g_{2}$ is convex in the direction of the real axis.

Remark 6. The convolution of $l_{0}$, which is the right half-plane mapping presented in [2], with itself sends $\mathbb{D}$ onto a nonconvex domain. This is because

$$
h_{0}(z) * h_{0}(z)=z+\sum_{n=2}^{\infty} \frac{(1+n)^{2}}{4} z^{n} \notin C .
$$

Hence by Theorem 5.7 in $[2], l_{0} \widetilde{*} l_{0} \notin K_{H}^{O}$. Thus the conclusion of Theorem 5 cannot be strengthened to $f_{1} \widetilde{*} f_{2} \in K_{H}^{O}$.

The next theorem shows that the harmonic convolution of a right half-plane mapping and a vertical strip mapping will also be convex in the real direction, provided that the convolution is sense-preserving.

Theorem 7. Let $f_{1}=h_{1}+\bar{g}_{1} \in K_{H}^{O}$ be a right half-plane mapping and $f_{2}=$ $h_{2}+\bar{g}_{2} \in K_{H}^{O}$ be a vertical strip mapping. If $f_{1} \widetilde{*} f_{2}$ satisfies (1), then $f_{1} \widetilde{*} f_{2} \in C_{H}^{O}$ and is convex in the direction of the real axis.

Proof. Let

$$
\begin{aligned}
& F_{1}=\left(h_{1}+g_{1}\right) *\left(h_{2}-g_{2}\right)=h_{1} * h_{2}-h_{1} * g_{2}+h_{2} * g_{1}-g_{1} * g_{2} \\
& F_{2}=\left(h_{1}-g_{1}\right) *\left(h_{2}+g_{2}\right)=h_{1} * h_{2}+h_{1} * g_{2}-h_{2} * g_{1}-g_{1} * g_{2},
\end{aligned}
$$

and so

$$
h_{1} * h_{2}-g_{1} * g_{2}=\frac{1}{2}\left[F_{1}+F_{2}\right] .
$$

As before, we want to show that this is a function convex in the direction of the real axis.

First, applying eq. (2) to $h_{2}+g_{2}$, we have

$$
\begin{aligned}
z F_{1}^{\prime} & =z\left[\left(h_{1}+g_{1}\right) *\left(h_{2}-g_{2}\right)\right]^{\prime} \\
& =\left(h_{1}+g_{1}\right) * z\left(h_{2}^{\prime}-g_{2}^{\prime}\right) \\
& =\left(h_{1}+g_{1}\right) *\left[z\left(h_{2}^{\prime}+g_{2}^{\prime}\right)\left(\frac{h_{2}^{\prime}-g_{2}^{\prime}}{h_{2}^{\prime}+g_{2}^{\prime}}\right)\right] \\
& =\frac{z}{1-z} * \frac{z}{\left(1+z e^{i \alpha}\right)\left(1+z e^{-i \alpha}\right)}\left(\frac{1-\omega}{1+\omega}\right) \\
& =\frac{z p_{1}(z)}{\left(1+z e^{i \alpha}\right)\left(1+z e^{-i \alpha}\right)},
\end{aligned}
$$


where $\operatorname{Re}\left\{p_{1}(z)\right\}>0, \forall z \in \mathbb{D}$. Hence,

$$
\operatorname{Re}\left\{\frac{z F_{1}^{\prime}}{\frac{z}{\left(1+z e^{i \alpha}\right)\left(1+z e^{-i \alpha}\right)}}\right\}>0 .
$$

Next, consider

$$
\begin{aligned}
z F_{2}^{\prime} & =z\left[\left(h_{1}-g_{1}\right) *\left(h_{2}+g_{2}\right)\right]^{\prime} \\
& =z\left(h_{1}^{\prime}-g_{1}^{\prime}\right) *\left(h_{2}+g_{2}\right) \\
& =\left[z\left(h_{1}^{\prime}+g_{1}^{\prime}\right)\left(\frac{h_{1}^{\prime}-g_{1}^{\prime}}{h_{1}^{\prime}+g_{1}^{\prime}}\right)\right] *\left(h_{2}+g_{2}\right) \\
& =\frac{z p_{2}(z)}{(1-z)^{2}} *\left(h_{2}+g_{2}\right),
\end{aligned}
$$

where $\operatorname{Re}\left\{p_{2}(z)\right\}>0, \forall z \in \mathbb{D}$. Using the fact that $\Psi(z) * z /(1-z)^{2}=z \Psi^{\prime}(z)$ and since $h_{2}+g_{2}$ is convex by eq. (2) we can apply Theorem 3 , we have

$$
\begin{aligned}
\operatorname{Re}\left\{\frac{z F_{2}^{\prime}}{\frac{z}{\left(1+z e^{i \alpha}\right)\left(1+z e^{-i \alpha}\right)}}\right\} & =\operatorname{Re}\left\{\frac{\left(h_{2}+g_{2}\right) * p_{2}(z) \frac{z}{(1-z)^{2}}}{z\left(h_{2}^{\prime}+g_{2}^{\prime}\right)}\right\} \\
& =\operatorname{Re}\left\{\frac{\left(h_{2}+g_{2}\right) * p_{2}(z) \frac{z}{(1-z)^{2}}}{\left(h_{2}+g_{2}\right) * \frac{z}{(1-z)^{2}}}\right\}>0 .
\end{aligned}
$$

Therefore, from eqs. (5) and (6),

$$
\operatorname{Re}\left\{\frac{z\left(F_{1}^{\prime}+F_{2}^{\prime}\right)}{\frac{z}{\left(1+z e^{i \alpha}\right)\left(1+z e^{-i \alpha}\right)}}\right\}>0
$$

and $F_{1}+F_{2}$ is convex in the direction of the real axis by Theorem 2. Finally, by applying Theorem 1 to eq. (4), we get the desired result.

Remark 8. Actually, we have proved a more general result since we let $f_{2}$ be any mapping in $K_{H}^{O}$ satisfying eq. (2). Hence $f_{2}$ may map $\mathbb{D}$ onto a vertical strip, a halfstrip, a trapezium, or a triangle. The same is true of the next theorem.

Theorem 9. Let $f=h+\bar{g} \in K_{H}^{O}$ be a vertical strip mapping. If $f \widetilde{*} f$ satisfies (1) and if $\operatorname{Re}\left\{(1-z)^{2}\left(h^{\prime}-g^{\prime}\right)\right\}>0, \forall z \in \mathbb{D}$, then $f \widetilde{*} f \in C_{H}^{O}$ and is convex in the direction of the real axis.

Proof. Let

$$
F=(h+g) *(h-g)=h * h-g * g .
$$

Notice that

$$
\begin{aligned}
z F^{\prime} & =z[(h+g) *(h-g)]^{\prime} \\
& =(h+g) * z\left(h^{\prime}-g^{\prime}\right) \\
& =(h+g) * \frac{z\left[(1-z)^{2}\left(h^{\prime}-g^{\prime}\right)\right]}{(1-z)^{2}} .
\end{aligned}
$$

Since we assumed that $\operatorname{Re}\left\{(1-z)^{2}\left(h^{\prime}-g^{\prime}\right)\right\}>0$, we can apply Theorem 3 to derive

$$
\operatorname{Re}\left\{\frac{z F^{\prime}}{z\left(h^{\prime}+g^{\prime}\right)}\right\}=\operatorname{Re}\left\{\frac{(h+g) * \frac{z}{(1-z)^{2}}\left[(1-z)^{2}\left(h^{\prime}-g^{\prime}\right)\right]}{(h+g) * \frac{z}{(1-z)^{2}}}\right\}>0 .
$$


Because

$$
z\left(h^{\prime}+g^{\prime}\right)=\frac{z}{\left(1+z e^{i \alpha}\right)\left(1+z e^{-i \alpha}\right)},
$$

Theorem 2 guarantees that $F$ is convex in the direction of the real axis, and the result follows by Theorem 1 .

Question 10. These results concern unbounded domains. In Example 4, it is stated that the harmonic convolution of a half-plane mapping with a 6-gon mapping is not in $K_{H}^{O}$. However, it is true that the harmonic convolution of that 6 -gon mapping with itself is in $K_{H}^{O}$. Does this work in general? That is, if $f \in K_{H}$ maps $\mathbb{D}$ onto a bounded domain, is $f \widetilde{*} f \in K_{H}$ ?

\section{REFERENCES}

[1] Abu-Muhanna, Y. and G. Schober, Harmonic mappings onto convex domains, Can. J. Math. 39, no. 6, (1987), 1489-1530.

[2] Clunie, J. and T. Sheil-Small, Harmonic univalent functions, Ann. Acad. Sci. Fenn. Ser. A.I Math. 9, (1984), 3-25.

[3] Dorff, M. J., Harmonic mappings onto asymmetric vertical strips, in Computational Methods and Function Theory 1997, (N. Papamichael, St. Ruscheweyh and E. B. Saff, eds.), 171-175, World Sci. Publishing, River Edge, NJ, 1999.

[4] Dorff, M. J., Some harmonic $n$-slit mappings, Proc. of the Amer. Math. Soc. 126 (1998), 569-576.

[5] Duren, P., A survey of harmonic mappings in the plane, Texas Tech. Univ., Math. Series, Visiting Scholars Lectures, 1990-1992 18 (1992), 1-15.

[6] Hengartner, W. and G. Schober, Univalent harmonic functions, Trans. Amer. Math. Soc. 299 (1987), 1-31.

[7] Pommerenke, C., On starlike and close-to-convex functions, Proc. London Math. Soc. (3) 13, (1963), 290-304.

[8] Ruscheweyh, St. and L. Salinas, On the preservation of direction-convexity and the GoodmanSaff conjecture, Ann. Acad. Sci. Fenn., Ser. A. I. Math. 14 (1989), 63-73.

[9] Ruscheweyh, St. and T. Sheil-Small, Hadamard products of schlicht functions and the PolyaSchoenberg conjecture, Comment. Math. Helv. 48 (1973), 119-135.

Department of Mathematics and Statistics, University of Missouri-Rolla, Rolla, MO 65409

E-mail address: mdorff@umr.edu 